\title{
Body mass index, abdominal fatness, and the risk of sudden cardiac death: a systematic review and dose-response meta-analysis of prospective studies
}

\author{
Dagfinn Aune ${ }^{1,2,3}$ (i) $\cdot$ Sabrina Schlesinger ${ }^{4} \cdot$ Teresa Norat $^{1} \cdot$ Elio Riboli $^{1}$
}

Received: 10 August 2017 / Accepted: 26 December 2017/ Published online: 7 February 2018

(C) The Author(s) 2018. This article is an open access publication

\begin{abstract}
Although overweight and obesity are established risk factors for some types of heart disease including ischemic heart disease, heart failure and atrial fibrillation, less is known about the association between adiposity and sudden cardiac death. We conducted a systematic review and meta-analysis of prospective studies to clarify the association between adiposity and risk of sudden cardiac death. PubMed and Embase databases were searched up to July 20th 2017. Summary relative risks (RRs) and 95\% confidence intervals (CIs) were calculated using random effects models. The summary RR was 1.16 $\left(95 \%\right.$ CI $\left.1.05-1.28, \mathrm{I}^{2}=68 \%, \mathrm{n}=14\right)$ per 5 unit increment in BMI, and $1.82\left(95 \% \mathrm{CI} 1.61-2.07, \mathrm{I}^{2}=0 \%, \mathrm{n}=3\right)$ per 0.1 unit increase in waist-to-hip ratio, and $1.03\left(95 \%\right.$ CI $\left.0.93-1.15, \mathrm{I}^{2}=0 \%, \mathrm{n}=2\right)$ per $10 \mathrm{~cm}$ increase in waist circumference. The heterogeneity in the analysis of BMI and sudden cardiac death persisted across most subgroup analyses. The association was stronger among studies with longer follow-up compared to short follow-up and was observed in the European and American studies, but not in the Asian studies. There was a J-shaped association between BMI and sudden cardiac death and the lowest risk was observed in the normal weight range, however, the increased risk with a low BMI was attenuated among studies with a longer duration of follow-up. This meta-analysis suggest an increased risk of sudden cardiac death with increasing BMI and waist-to-hip ratio, however, further studies with stratification for smoking status are needed of waist circumference, weight changes and adiposity at younger ages.
\end{abstract}

Keywords Obesity $\cdot$ BMI $\cdot$ Waist-to-hip ratio $\cdot$ Sudden cardiac death $\cdot$ Meta-analysis

Electronic supplementary material The online version of this article (https://doi.org/10.1007/s10654-017-0353-9) contains supplementary material, which is available to authorized users.

Dagfinn Aune

d.aune@imperial.ac.uk

1 Department of Epidemiology and Biostatistics, School of Public Health, Imperial College London, St. Mary's Campus, Norfolk Place, Paddington, London W2 1PG, UK

2 Bjørknes University College, Oslo, Norway

3 Department of Endocrinology, Morbid Obesity and Preventive Medicine, Oslo University Hospital, Oslo, Norway

4 Institute for Biometrics and Epidemiology, German Diabetes Center (DDZ), Heinrich Heine University Düsseldorf, Düsseldorf, Germany

\section{Introduction}

The prevalence of overweight and obesity has increased rapidly over the last decades in all areas of the world [1]. Overweight and obesity are important risk factors for a wide range of chronic diseases, including cardiovascular diseases, type 2 diabetes, several types of cancer as well as all-cause mortality [2-10], and the current trends are a major challenge for public health both in terms of reduced quality of life and increased medical costs [11].

Sudden cardiac death accounted for more than 350,000 deaths in the USA in 2014 [12]. Although clinical guidelines focus on reducing risk in high-risk patients using medical therapies, up to $75 \%$ of all sudden cardiac deaths occur in patients not classified as high-risk by current guidelines [13]. Although a substantial amount of data has consistently showed that overweight and obesity increases the risk of coronary heart disease [5], heart failure [6] and atrial fibrillation [14], all risk factors for sudden cardiac 
death, data are more limited and less consistent regarding the association between overweight and obesity and the risk of sudden cardiac death [15-27]. Some studies showed an increase in risk of sudden cardiac death with a greater body mass index (BMI, weight in $\mathrm{kg}$ divided by height squared in metres, $\mathrm{kg} / \mathrm{m}^{2}$ ) $[16,25,27]$, however, other studies found associations only in non-current smokers [26], no significant association [15, 20, 21], or even some increase in risk with a low BMI [18]. Given the increased prevalence of overweight and obesity globally establishing whether excess BMI also is related to increased risk of sudden cardiac death is of major public health importance and could inform guidelines for prevention. For these reasons we conducted a systematic review and meta-analysis of prospective studies of adiposity and the risk of sudden cardiac death. We aimed to clarify the direction and strength of the association, shape of the dose-response relationship and potential sources of heterogeneity between studies.

\section{Methods}

\section{Search strategy and inclusion criteria}

We searched the PubMed and Embase databases up to July 20th 2017 for eligible studies (DA, and SS). A list of the search terms used are provided in Supplementary Table 1 and 2. We followed standard criteria for reporting of metaanalyses [28]. In addition, we searched the reference lists of the relevant publications for further studies. Study quality was assessed using the Newcastle-Ottawa scale [29].

\section{Study selection}

We included prospective and retrospective cohort studies and nested case-control studies of the association between adiposity measures (BMI, waist circumference, waist-tohip ratio, hip circumference, and weight gain) and risk of sudden cardiac death that were published in English. Studies in high-risk populations (patient populations), abstract only publications, grey literature and unpublished studies were excluded. Adjusted relative risk estimates (hazard ratio, risk ratio, or odds ratio) had to be available with the $95 \%$ confidence intervals (CIs) in the publication and for the dose-response analysis, a quantitative measure of adiposity and the total number of cases and person-years or non-cases had to be available in the publication. When multiple publications were available from the same study we used the study with the largest number of cases. A list of the excluded studies and exclusion reasons are found in the Supplementary Table 3.

\section{Data extraction}

We extracted the following data from each study: The first author's last name, publication year, country where the study was conducted, study period, sample size, number of cases/controls, exposure variable, exposure level, relative risks and $95 \%$ confidence intervals for the highest versus the lowest level of the exposure variable and variables adjusted for in the analysis. Data were extracted by one reviewer (DA) and checked for accuracy by a second reviewer (SS). Any disagreements were resolved through discussion.

\section{Statistical analysis}

We calculated summary RRs and $95 \%$ CIs for a 5 unit increment in BMI, a 0.1 unit increment in waist-to-hip ratio, and $10 \mathrm{~cm}$ increase in waist circumference (approximately equal to one standard deviation for each measure) using a random effects model, which takes into account heterogeneity between studies [30]. For the primary analysis we used the model from each study that had the greatest degree of control for potential confounding with the exception of when potential intermediate risk factors were adjusted for in a separate step (as an exploration of how much of the association might be mediated by cholesterol for example). The average of the natural logarithm of the RRs was estimated and the RR from each study was weighted according to the method of DerSimonian and Laird [30]. A two-tailed $p<0.05$ was considered statistically significant. If studies reported results separately for men and women or other subgroups we combined the subgroup-specific estimates using a fixed-effects model to generate an overall estimate so that each study was only represented once in the main analysis, but sex-specific results are presented separately in subgroup analyses.

The method described by Greenland and Longnecker [31] was used for the dose-response analysis of adiposity measures and we calculated study-specific slopes (linear trends) and 95\% CIs from the natural logs of the reported RRs and CIs across categories of each adiposity measures. The mean level of BMI or WHR in each category was assigned to the corresponding relative risk for each study and for studies that reported the exposures in ranges we calculated the average of the upper and the lower cut-off point which was used as a midpoint. When the lowest or highest category was open-ended or had an extreme range we used the width of the adjacent interval to calculate an upper or lower cut-off value. For studies that reported continuous risk estimates per $1 \mathrm{BMI}$ unit or per $3.3 \mathrm{BMI}$ units these risk estimates were converted to a risk estimate per 5 BMI units by first taking the natural logarithm of the 
RR $(95 \% \mathrm{CI})$, then dividing the $\ln (\mathrm{RR}, 95 \% \mathrm{CI})$ by the increment reported, then multiplying by 5 , and backtransforming to non-logarithmic scale before inclusion in the meta-analysis. A potential nonlinear dose-response relationship between BMI, waist-to-hip ratio, and waist circumference and risk of sudden cardiac death was examined by using fractional polynomial models [32]. We determined the best fitting second order fractional polynomial regression model, defined as the one with the lowest deviance. A likelihood ratio test was used to assess the difference between the nonlinear and linear models to test for nonlinearity [32]. Studies that only reported a continuous risk estimate and not categorical data were excluded in the nonlinear dose-response analysis as it requires that data are reported for at least three categories of BMI.

Subgroup analyses stratified by sex, measurement versus self-report of adiposity measures, duration of follow-up, geographic location, number of cases, study quality scores, and adjustment for confounders (age, smoking, alcohol, physical activity) and potential intermediates (hypertension, blood pressure, cholesterol, diabetes mellitus, coronary heart disease, heart failure, and left ventricular hypertrophy) were conducted to investigate potential sources of heterogeneity and heterogeneity between studies was quantitatively assessed by the $\mathrm{Q}$ test and $\mathrm{I}^{2}$ [33]. Metaregression analyses were used to examine between subgroup differences in the summary estimates. Small study effects, such as publication bias, were assessed by inspecting the funnel plots for asymmetry and with Egger's test [34] and Begg's test [35] with the results considered to indicate small study effects when $p<0.10$. Sensitivity analyses excluding one study at a time were conducted to clarify whether the results were simply due to one large study or a study with an extreme result. The statistical analyses were conducted using Stata software version 13.0 (StataCorp, College Station, TX, USA).

\section{Results}

We identified 14 prospective studies (13 publications) [15-27] that were included in the systematic review of BMI, waist-to-hip ratio, and waist circumference and risk of sudden cardiac death (Supplementary Table 4; Fig. 1). Only one study reported on BMI in early adulthood (at age 18 years) [25] or weight change [25] and sudden cardiac death and meta-analyses were therefore not possible for these measures. All studies reported on BMI at baseline and the studies were conducted mostly in middle-aged populations. The age range or mean age for each study is provided in Supplementary Table 4 and the lower and higher age range across studies was 30 and 84 years, respectively. The mean (median) duration of follow-up was

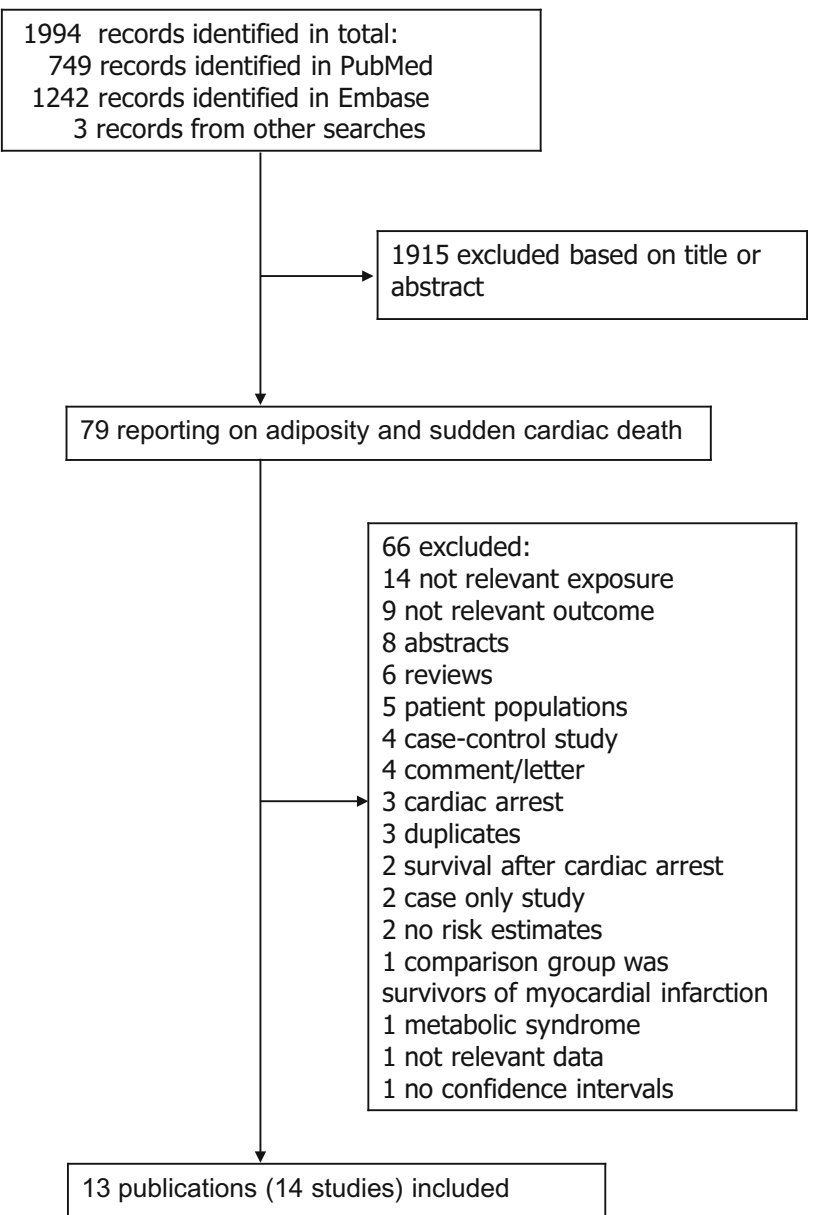

Fig. 1 Flow-chart of study selection

16.4 (11.8) years and ranged from 5.2 to 38 years. Three studies included only men, two included only women and nine studies included both men and women. Eight studies were from Europe, three were from the USA, and three were from Asia (Japan) (Supplementary Table 4).

\section{Body mass index}

Fourteen prospective studies (11 publications) [15-18, 20-22, 24-27] were included in the linear doseresponse analysis of BMI and sudden cardiac death incidence and included 3376 cases among 406,079 participants. The summary RR for a 5 unit increment in BMI was $1.16\left(95 \%\right.$ CI $1.05-1.28, \mathrm{I}^{2}=68.2 \%$, pheterogeneity $<$ 0.0001) (Fig. 2a). All but one of the studies found increased risk, but the strength of the association differed between studies. In sensitivity analyses excluding the most influential studies, the summary RR ranged from 1.14 (95\% CI 1.03-1.27) when excluding the Nurses' Health Study [25] to 1.20 (95\% CI 1.09-1.31) when excluding the Health 2002 study [22] (Supplementary Table 5). There was no indication of publication bias with Egger's test, $p=0.18$, 
Fig. 2 BMI and sudden cardiac death, linear and nonlinear dose-response analysis
A

Body mass index and sudden cardiac death, dose-response analysis, per 5 BMI units

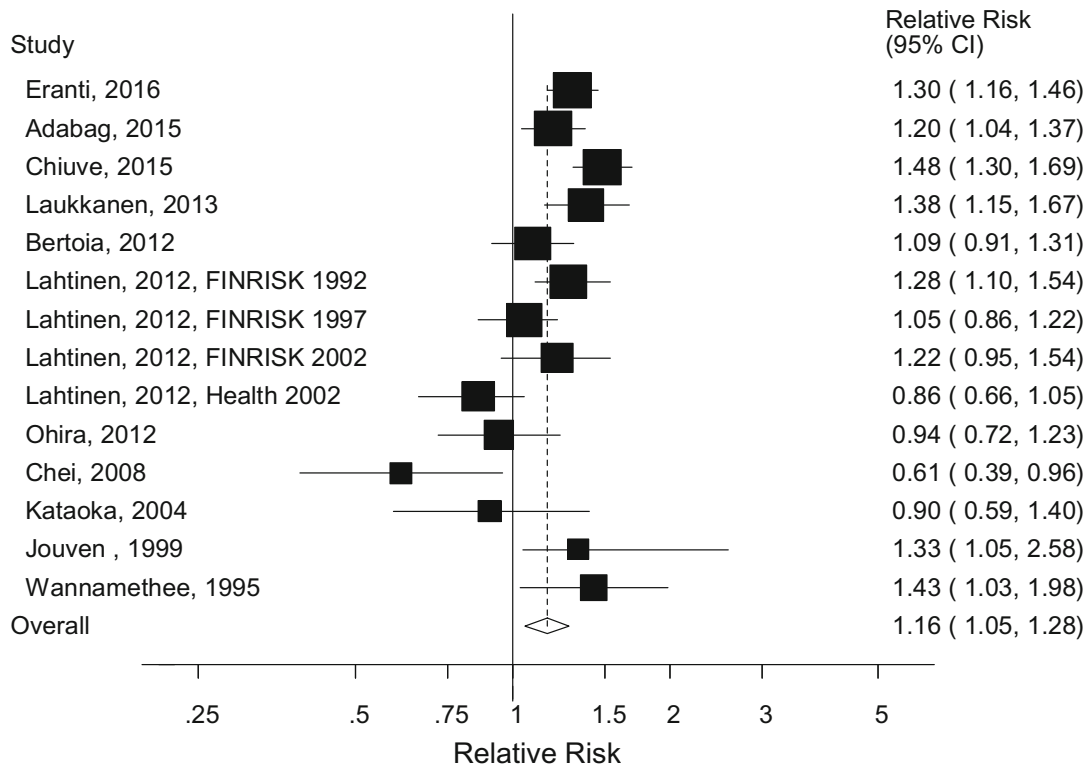

B

Body mass index and sudden cardiac death, nonlinear dose-response analysis

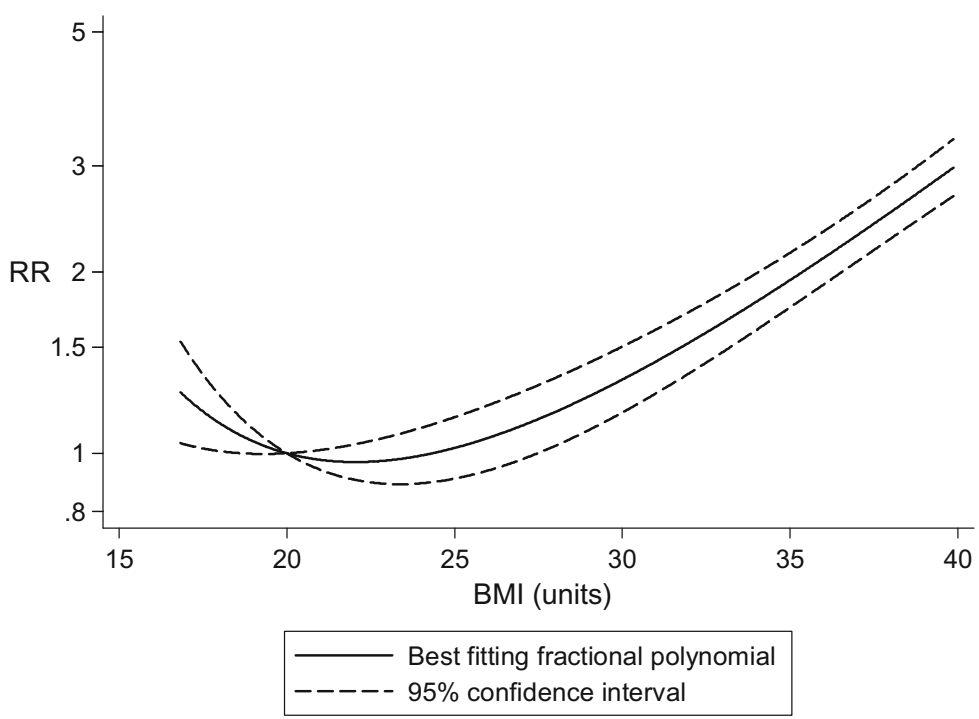

or with Begg's test, $p=0.27$, and there was no evidence of asymmetry by inspection of the funnel plot (Supplementary Figure 1). Seven studies [15, 18, 20, 21, 25-27] were included in the nonlinear dose-response analysis. There was evidence of a nonlinear J-shaped association between BMI and sudden cardiac death, p ponlinearity $<0.0001$ (Fig. 2b, Supplementary Table 6) with a slight increase in risk in the underweight categories, and a 14\%, 60\% and 2-3 fold increase in risk in the overweight, obese, and severely obese categories. When the nonlinear dose-response analysis was stratified by duration of follow-up the association between low BMI and increased risk of sudden cardiac death was stronger among the studies with $<10$ years follow-up than among studies with $\geq 10$ years follow-up, and in addition, the optimal BMI was around 23 and 20-22 among the studies with short and long-follow-up duration, respectively (Supplementary Figures 2, 3).

\section{Waist-to-hip ratio and waist circumference}

Three prospective studies [19, 21, 26] were included in the analysis of waist-to-hip ratio and risk of sudden cardiac 
Fig. 3 Waist-to-hip ratio and sudden cardiac death
A

Waist-to-hip ratio and sudden cardiac death, dose-response analysis, per 0.1 units

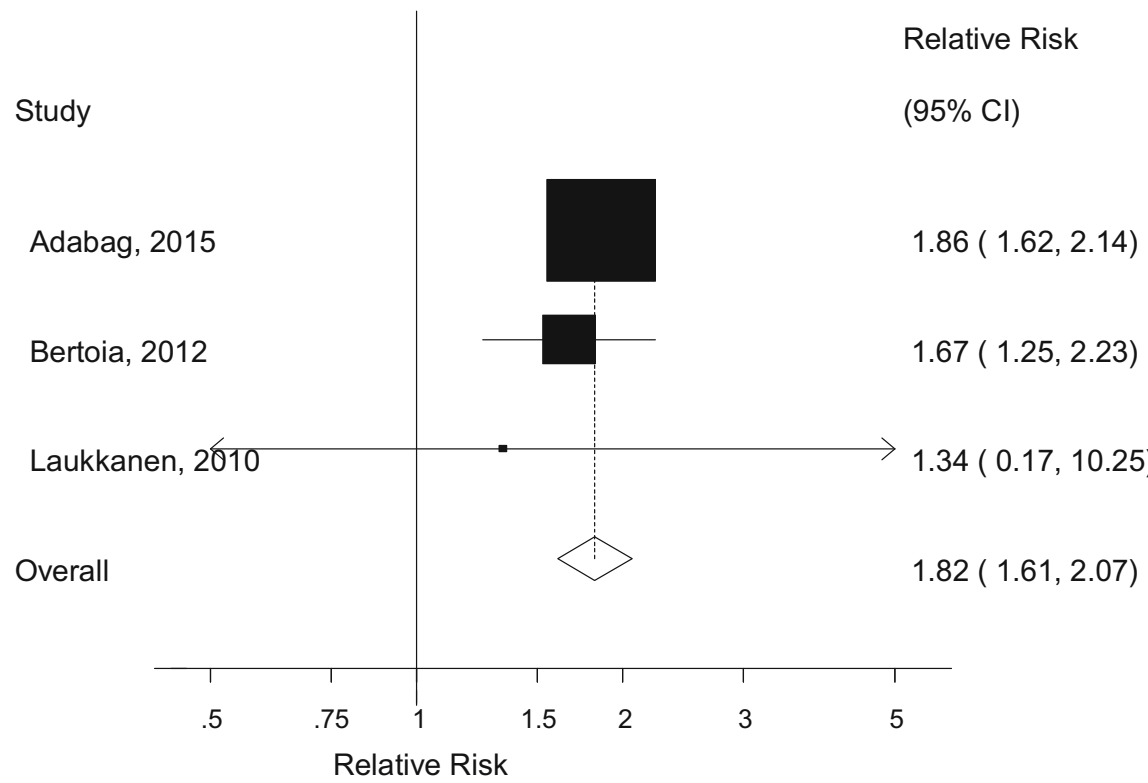

B

Waist-to-hip ratio and sudden cardiac death, nonlinear dose-response analysis

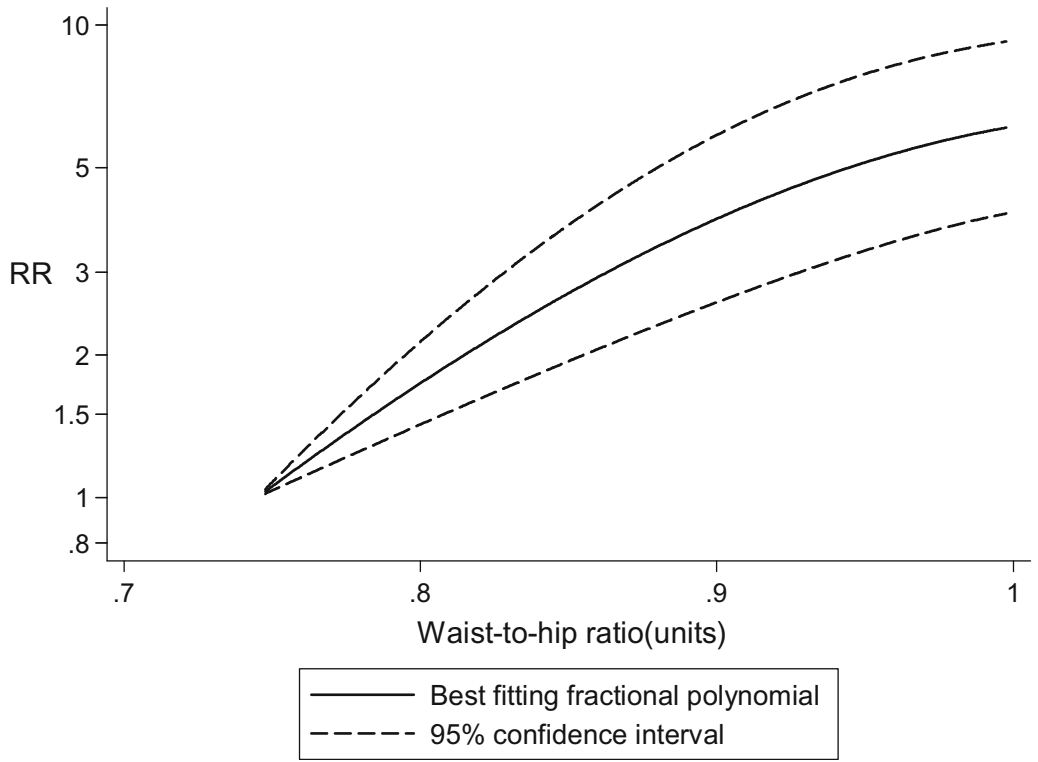

death (817 cases, 179,117 participants) and the summary RR for a 0.1 unit increment in waist-to-hip ratio was 1.82 $\left(95 \% \quad\right.$ CI $\left.1.61-2.07, \quad I^{2}=0 \%, \quad p_{\text {heterogeneity }}=0.77\right)$ (Fig. 3a). Although the test for nonlinearity was significant, $\mathrm{p}_{\text {nonlinearity }}=0.02$, there was no evidence of a threshold effect and the association increased strongly even with modest increases in waist-to-hip ratio (Fig. 3b, Supplementary Table 7).

Two prospective studies $[23,26]$ reported on waist circumference and sudden cardiac death (312 cases, 15,972 participants) and the summary RR for a $10 \mathrm{~cm}$ increase was $1.03\left(95 \%\right.$ CI $0.93-1.15, \mathrm{I}^{2}=0 \%$, pheterogeneity $=$ 0.54) (Fig. 4). Nonlinear dose-response analysis was not possible because only one of the studies reported categorical data. 
Fig. 4 Waist circumference and sudden cardiac death, per $10 \mathrm{~cm}$

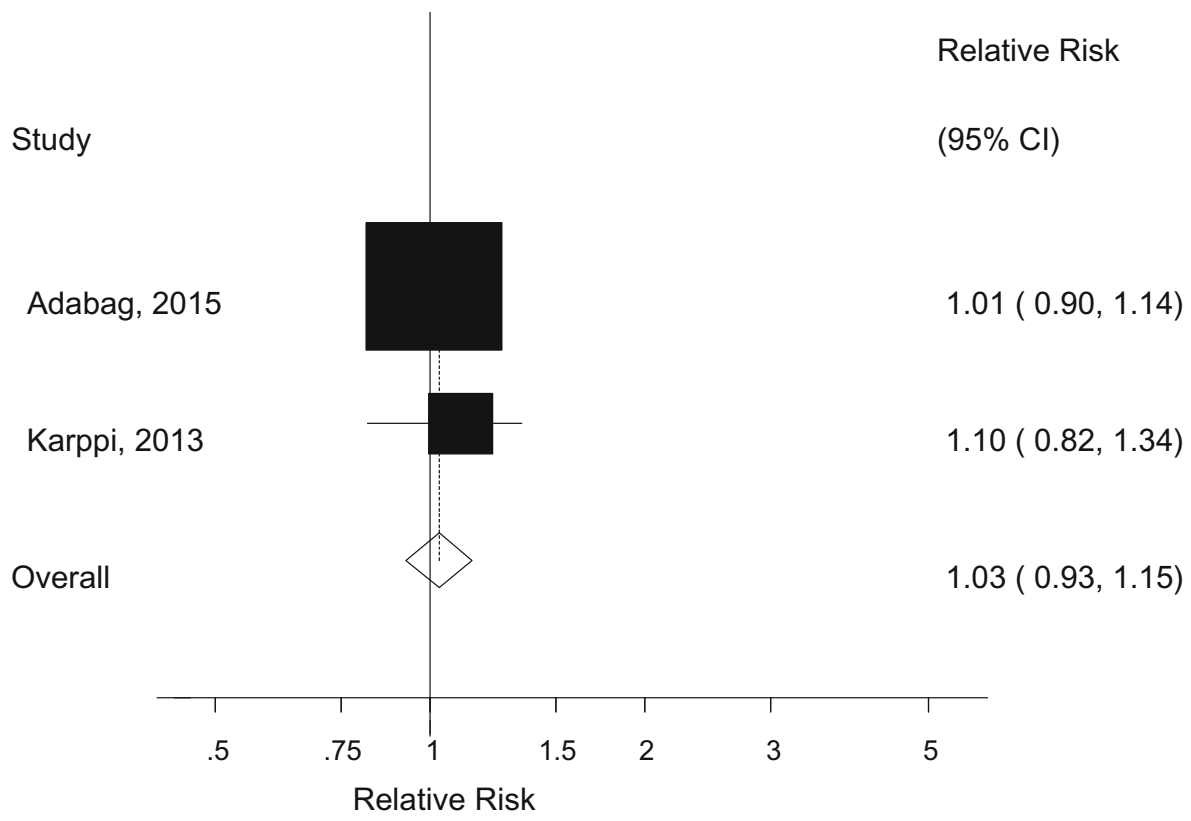

\section{Subgroup and sensitivity analyses and study quality}

In subgroup analyses of the association between BMI and sudden cardiac death there was suggestive heterogeneity when the analyses were stratified by duration of follow-up, $p_{\text {heterogeneity }}=0.06$, with stronger associations among studies with longer duration of follow-up, and when analyses were stratified by geographic location, pheterogeneity $=$ 0.09 , with no association among the Asian studies, while a positive association was observed among the European and North American studies (Table 1). There was no significant heterogeneity between the remaining subgroup analyses and there were positive associations in most of them although the association was not always significant possibly because of the limited number of studies in some subgroups. Study quality was high with a mean (median) score of 7.6 [8] out of 9 points (Supplementary Table 8).

\section{Discussion}

This is to our knowledge is the first meta-analysis of adiposity and the risk of sudden cardiac death. There was a $16 \%$ increase in the relative risk per 5 units increase in $\mathrm{BMI}$ and an $82 \%$ increase in relative risk per 0.1 unit increase in waist-to-hip ratio, based on eight and three studies, respectively, however, no association was observed among two studies of waist circumference. There was evidence of a nonlinear J-shaped association between BMI and sudden cardiac death, with a slight increase in risk in the underweight categories, and a $14 \%, 60 \%$ and 2-3 fold increase in risk in the overweight, obese and severely obese categories. The association between waistto-hip ratio and sudden cardiac death was also nonlinear, but the dose-response curve was slightly steeper at lower levels of waist-to-hip ratio than at higher levels. Our current findings are consistent with previous studies that have found that adiposity increases the risk of other heart conditions that increase the risk of sudden cardiac death including coronary heart disease [5], heart failure [6], and atrial fibrillation [14].

Our meta-analysis has some limitations that need to be mentioned. Confounding by other risk factors may have influenced the results. We conducted several subgroup analyses to try to clarify whether adjustment for specific confounding factors influenced the summary estimates. The association between BMI and sudden cardiac death was in the direction of increased risk in most subgroup analyses, however, it was not significant in every subgroup analysis (possibly because there were few studies and low statistical power in some subgroups). More importantly, there was no heterogeneity between the subgroups analyses stratified by adjustment for confounding factors and potential intermediate factors. The association was not significant among the studies that adjusted for hypertension (which could be considered an intermediate factor), however, the association was significant among the studies that adjusted for blood pressure. The reason for the difference might be a mix of chance and limited statistical power in the analysis with adjustment for hypertension as there were only four studies in that subgroup analysis, while there were eight studies in the subgroup analysis with adjustment 
Table 1 Subgroup analyses of BMI and sudden cardiac death

\begin{tabular}{|c|c|c|c|c|c|}
\hline & \multicolumn{5}{|c|}{ BMI } \\
\hline & $n$ & RR $(95 \%$ CI $)$ & $\mathrm{I}^{2}(\%)$ & $P_{\mathrm{h}}^{\mathrm{a}}$ & $P_{\mathrm{h}}^{\mathrm{b}}$ \\
\hline All studies & 14 & $1.16(1.05-1.28)$ & 68.2 & $<0.0001$ & \\
\hline \multicolumn{6}{|l|}{ Sex } \\
\hline Men & 3 & $1.11(0.70-1.75)$ & 70.7 & 0.03 & \multirow[t]{3}{*}{$0.82 / 0.99^{\circ}$} \\
\hline Women & 3 & $1.16(0.83-1.62)$ & 82.8 & 0.003 & \\
\hline Men and women & 9 & $1.15(1.04-1.27)$ & 59.2 & 0.01 & \\
\hline \multicolumn{6}{|l|}{ Assessment of weight/height } \\
\hline Measured & 10 & $1.19(1.10-1.30)$ & 47.8 & 0.05 & \multirow[t]{3}{*}{0.21} \\
\hline Self-reported (validated) & 2 & $0.98(0.41-2.33)$ & 92.8 & $<0.0001$ & \\
\hline Not available & 2 & $0.93(0.74-1.17)$ & 0 & 0.88 & \\
\hline \multicolumn{6}{|l|}{ Duration of follow-up } \\
\hline$<5$ years & 0 & & & & \multirow[t]{5}{*}{0.06} \\
\hline $5-<10$ years & 5 & $0.99(0.76-1.28)$ & 70.6 & 0.009 & \\
\hline $10-<15$ years & 3 & $1.12(1.02-1.23)$ & 0 & 0.49 & \\
\hline $15-<20$ years & 2 & $1.32(1.17-1.50)$ & 0 & 0.54 & \\
\hline$\geq 20$ years & 4 & $1.28(1.08-1.51)$ & 67.8 & 0.03 & \\
\hline \multicolumn{6}{|l|}{ Geographic location } \\
\hline Europe & 8 & $1.21(1.08-1.35)$ & 55.8 & 0.03 & \multirow[t]{3}{*}{0.09} \\
\hline America & 3 & $1.26(1.05-1.50)$ & 77.7 & 0.01 & \\
\hline Asia & 3 & $0.84(0.66-1.07)$ & 26.3 & 0.26 & \\
\hline \multicolumn{6}{|l|}{ Number of cases } \\
\hline Cases $<100$ & 2 & $0.89(0.45-1.73)$ & 85.8 & 0.008 & \multirow[t]{3}{*}{0.44} \\
\hline Cases $100-<200$ & 6 & $1.18(1.01-1.39)$ & 64.3 & 0.02 & \\
\hline Cases $\geq 200$ & 6 & $1.20(1.05-1.36)$ & 69.4 & 0.006 & \\
\hline \multicolumn{6}{|l|}{ Study quality } \\
\hline $0-3$ & 0 & & & & \multirow[t]{3}{*}{0.35} \\
\hline $4-6$ & 1 & $0.94(0.72-1.23)$ & & & \\
\hline $7-9$ & 13 & $1.18(1.07-1.30)$ & 67.7 & $<0.0001$ & \\
\hline \multicolumn{6}{|l|}{ Adjustment for confounders } \\
\hline \multicolumn{6}{|l|}{ Age } \\
\hline Yes & 14 & $1.16(1.05-1.28)$ & 68.2 & $<0.0001$ & \multirow[t]{2}{*}{$\mathrm{NC}$} \\
\hline No & 0 & & & & \\
\hline \multicolumn{6}{|l|}{ Smoking } \\
\hline Yes & 12 & $1.14(1.02-1.28)$ & 72.4 & $<0.0001$ & \multirow[t]{2}{*}{0.49} \\
\hline No & 2 & $1.23(1.08-1.40)$ & 0 & 0.32 & \\
\hline \multicolumn{6}{|l|}{ Alcohol } \\
\hline Yes & 4 & $1.11(0.82-1.48)$ & 85.6 & $<0.0001$ & \multirow[t]{2}{*}{0.99} \\
\hline No & 10 & $1.16(1.07-1.27)$ & 45.7 & 0.06 & \\
\hline \multicolumn{6}{|l|}{ Physical activity } \\
\hline Yes & 6 & $1.09(0.89-1.34)$ & 83.2 & $<0.0001$ & \multirow[t]{2}{*}{0.54} \\
\hline No & 8 & $1.21(1.10-1.33)$ & 36.7 & 0.14 & \\
\hline Adjustment for potential in & & & & & \\
\hline Hypertension & & & & & \\
\hline Yes & 4 & $1.03(0.76-1.40)$ & 87.0 & $<0.0001$ & 0.48 \\
\hline No & 10 & $1.19(1.09-1.31)$ & 49.3 & 0.04 & \\
\hline Blood pressure & & & & & \\
\hline Yes & 8 & $1.17(1.05-1.32)$ & 58.1 & 0.02 & 0.89 \\
\hline No & 6 & $1.13(0.94-1.37)$ & 79.2 & $<0.0001$ & \\
\hline
\end{tabular}


Table 1 (continued)

\begin{tabular}{|c|c|c|c|c|c|}
\hline & \multicolumn{5}{|c|}{ BMI } \\
\hline & $n$ & RR $(95 \%$ CI $)$ & $\mathrm{I}^{2}(\%)$ & $P_{\mathrm{h}}^{\mathrm{a}}$ & $P_{\mathrm{h}}^{\mathrm{b}}$ \\
\hline \multicolumn{6}{|c|}{ Cholesterol } \\
\hline Yes & 6 & $1.27(1.11-1.45)$ & 60.1 & 0.03 & 0.24 \\
\hline No & 8 & $1.10(0.97-1.24)$ & 62.0 & 0.01 & \\
\hline \multicolumn{6}{|c|}{ Diabetes mellitus } \\
\hline Yes & 13 & $1.11(0.98-1.26)$ & 73.6 & $<0.0001$ & 0.24 \\
\hline No & 3 & $1.28(1.15-1.42)$ & 0 & 0.37 & \\
\hline \multicolumn{6}{|c|}{ Coronary heart disease } \\
\hline Yes & 7 & $1.17(1.05-1.30)$ & 62.4 & 0.01 & 0.90 \\
\hline No & 7 & $1.13(0.93-1.37)$ & 75.2 & $<0.0001$ & \\
\hline
\end{tabular}

$n$ denotes the number of studies. The number of studies is not always equal to the total because the subgroup analyses were not applicable to some studies or information was not provided in the publication

${ }^{\mathrm{a}} \mathrm{P}$ for heterogeneity within each subgroup

${ }^{\mathrm{b}} \mathrm{P}$ for heterogeneity between subgroups

${ }^{\mathrm{c}} \mathrm{P}$ for heterogeneity between men and women (excluding men/women combined)

for blood pressure. The association persisted among studies that adjusted for serum cholesterol and coronary heart disease, but not among studies that adjusted for diabetes mellitus, but again there was no heterogeneity between the subgroups.

There was suggestion of a stronger association between BMI and sudden cardiac death among studies with longer compared to shorter durations of follow-up. There was no association among the studies with $5-<10$ years of followup, but a $12 \%$ increase in the relative risk per 5 BMI units among studies with $10<15$ years follow-up and a $32 \%$ and $28 \%$ increase in the relative risk among studies with $15-<20$ and $\geq 20$ years follow-up, respectively. The increased risk observed with a low BMI in the nonlinear dose-response analysis was also substantially attenuated among studies with longer ( $\geq 10$ years) follow-up, while it was stronger among the studies with shorter $(<10$ years) follow-up and the optimal BMI was around 23 among the studies with short follow-up, and around 20-22 among the studies with longer follow-up. This is similar to what we have previously found in relation to heart failure [6] and all-cause mortality [4] and might to some degree reflect confounding by illness and associated weight loss which may have a greater impact in studies with a short duration of follow-up than among studies with a long duration of follow-up [4, 6], or alternatively it might reflect weight gain over time which could contribute to increased risk beyond that of baseline BMI [25]. For example, if only a baseline anthropometric assessment was conducted in the included studies, people who were normal weight at baseline might become overweight, and people who were overweight might become obese (given trends with increased weight gain in most populations over time [1]), but because of only the baseline assessment they would still be categorized as normal and overweight, respectively. The studies with the longest duration of follow-up would then also be the studies were the weight increased the most over time and if weight gain increases risk of sudden cardiac death, those studies would show the strongest associations. Lastly, smoking may be a strong confounding factor of the association between BMI and health outcomes because smoking is strongly associated with a number of chronic diseases and mortality [36], but at the same time is associated with lower BMI [37] and may therefore confound and drive the optimal BMI upwards as observed in our previous analysis of BMI and all-cause mortality [4] and might also at least partly explain the observed slight increase in risk of sudden cardiac death with a low BMI of 16.7-17.5 compared to a BMI of around 20-22. Unfortunately it was not possible to conduct similar stratified analyses by smoking status in the current meta-analysis because only one of the included studies reported such stratified analyses [26]. However, that study reported RRs (95\% CIs) of 1.73 (1.06-2.84), 1.94 (1.12-3.33), and 3.36 (1.86-6.07) for BMI categories of 25-29.9, $30-34.9, \geq 35.0$ compared to $18.5-24.9$ among nonsmokers and $1.39(0.43-4.50), 0.86 \quad(0.55-1.33,0.94$ $(0.53-1.65), \quad 0.34 \quad(0.08-1.41)$ for BMI categories of $<18.5$, and $25-29.9,30-34.9, \geq 35.0$ compared to 18.5-24.9 among current smokers, strongly supporting a negative confounding effect of smoking on the association between BMI and sudden cardiac death.

Measurements of weight, height, waist and hip circumferences may have been affected by measurement errors, however, the association for BMI was significant only among the studies that used measured weight and height, 
not among those that used self-reported weight and height or those where the assessment of weight and height was unclear. This is probably also a chance finding as there was only two studies with either self-reported or unclear anthropometric measurements and because there was no heterogeneity between the different measurements of anthropometric factors. Validation studies have reported high correlations between self-reported and measured anthropometric measures [38-41]. BMI is an imperfect measure of body fatness as it does not distinguish between body fat and muscle mass. However, studies have shown high correlations between BMI and waist measures and body fat as measured by dual-energy $\mathrm{x}$-ray absorptiometry (DXA) [42, 43]. The association between adiposity and sudden cardiac death was positive for both BMI and waistto-hip ratio, although the dose-response relationship appeared to be stronger for the latter. Although publication bias or small study bias can affect the findings of metaanalyses of published literature, we found no evidence of such bias with Egger's or Begg's test. Because there was only one study on BMI in early adulthood and weight changes in relation to sudden cardiac death and because of the limited number of studies on waist circumference further studies are needed of these adiposity measures in relation to risk of sudden cardiac death.

Several mechanisms could explain an association between body fatness and increased risk of sudden cardiac death. Adiposity is associated metabolic disturbances including higher levels of total and LDL-cholesterol and lower HDL cholesterol [3], dyslipidemia [44], insulin resistance and diabetes [45] and inflammation which contributes to increased risk of sudden cardiac death [16, 46, 47]. Adiposity increases hemodynamic stress which activates the renin-antiotensin-aldosterone system leading to elevated aldosterone levels, which again increases blood volume and cardiac output and thereby contributes to left ventricular hypertrophy [48-51]. Adiposity also contributes to increased risk of hypertension [2] and higher resting heart rate $[52,53]$, which increases the risk of sudden cardiac death [22, 54]. Echocardiographic studies have shown that the heart adapts to obesity through eccentric cardiac hypertrophy, but to hypertension through concentric hypertrophy, and when both obesity and hypertension coexists, features of both concentric and eccentric ventricular hypertrophy results [55]. Obesity is associated with the secretion of cytokines that influence the heart's physiology and structure. High levels of leptin observed in obesity increases myocardial fat, reduces contractility and hypertrophy [56]. Adiposity is associated with low-grade inflammation and elevated levels of inflammatory markers such as tumor necrosis factor-alpha which reduces adiponectin and thereby increases left ventricular hypertrophy through AMP kinase signaling and alpha-adrenergic receptor stimulation [57]. Insulin resistance contributes to left ventricular hypertrophy through IGF-1 receptor stimulation [58]. Obesity is also associated with increased risk of cardiomyopathy [59], which increases the susceptibility to ventricular arrhytmia and sudden cardiac death. Excess weight is also associated with increased risk of medical conditions such as coronary heart disease [60], heart failure [6], and atrial fibrillation [14], which are established risk factors for sudden cardiac death [16, 46, 47, 61]. Obese patients without and with eccentric left ventricular hypertrophy have been shown to have 10-fold and 30-fold increased risk of premature ventricular contractions, respectively, compared to lean persons [62]. Ventricular ectopy (three or more premature beats per hour) or ventricular tachycardia are associated with increased risk of mortality among patients with coronary artery disease, valvular disease and cardiomyopathy and has been shown to be responsible for three out of four ventricular fibrillation episodes [63, 64]. Necropsy studies have found epicardial fat infiltration of the sinus node, fatty and fibrotic changes involving the atrioventricular node, and myocyte hypertrophy in morbidly obese subjects $[65,66]$. Studies have also found that obesity and morbid obesity is associated with alterations of the heart rate, pulse rate interval, QRS duration, QTc interval, P-, QRS-, T-wave axes and low QRS voltage [67, 68], which have been associated with increased risk of sudden cardiac death [69-72], and that weight loss by bariatric surgery reduced some of these alterations [73]. In one study the multivariable-adjusted hazard ratio for a BMI of $\geq 35$ versus 21.0-22.9 was 2.18 (95\% CI 1.44-3.28) and when further adjusted for potential intermediates, including high cholesterol, hypertension, diabetes, angina, and heart failure it was attenuated to 1.72 (95\% CI 1.13-2.60), suggesting that part of the association might be mediated by these traditional risk factors, but that other risk factors (e.g. left ventricular hypertrophy, resting heart rate and others) also could contribute to the increased risk.

Our meta-analysis has several strengths including the prospective design of the included studies which avoids recall bias and reduces the possibility for selection bias, and with $>3300$ cases and $>406,000$ participants there was sufficient statistical power to detect moderate associations. Additional strengths include the detailed dose-response analyses which clarified the shape of the doseresponse relationship and the robustness of the findings in multiple subgroup analyses as well as the high study quality of the included studies.

The findings have important clinical and public health implications because of the increasing prevalence of overweight and obesity worldwide [1], thus if current trends continue unabated it might contribute to an increased rates of sudden cardiac death. 
In conclusion, this meta-analysis suggest an increased risk of sudden cardiac death with increasing BMI and waist-to-hip ratio, however, further studies with adjustment for confounding factors and with stratified analyses by smoking status are needed of waist circumference, weight changes and adiposity at younger ages.

Acknowledgements This work has been supported by funding from the Imperial College National Institute of Health Research (NIHR) Biomedical Research Centre (BRC) and the South-East Regional Health Authorities of Norway. The study sponsor had no role in the study design, collection of data, analysis, and interpretation of data. D. Aune takes primary responsibility for the integrity of the data and the accuracy of the data analysis. We thank Darren C. Greenwood (Biostatistics Unit, Centre for Epidemiology and Biostatistics, University of Leeds, Leeds, United Kingdom) for providing the Stata code for the nonlinear dose-response analysis.

Open Access This article is distributed under the terms of the Creative Commons Attribution 4.0 International License (http://crea tivecommons.org/licenses/by/4.0/), which permits unrestricted use, distribution, and reproduction in any medium, provided you give appropriate credit to the original author(s) and the source, provide a link to the Creative Commons license, and indicate if changes were made.

Author's contributions DA: Conceived and designed the research. DA, SS: Acquired the data. DA, SS, TN, ER: Analyzed and interpreted the data. DA: Performed statistical analysis. ER, TN: Handled funding and supervision. DA: Drafted the manuscript. DA, SS, ER, TN: Made critical revision of the manuscript for intellectual content. DA, SS: Reference screening.

\section{References}

1. NCD Risk Factor Collaboration (NCD-RisC). Worldwide trends in body-mass index, underweight, overweight, and obesity from 1975 to 2016: a pooled analysis of 2416 population-based measurement studies in 128.9 million children, adolescents, and adults. Lancet 2017;390:2627-2642.

2. Guh DP, Zhang W, Bansback N, Amarsi Z, Birmingham CL, Anis $\mathrm{AH}$. The incidence of co-morbidities related to obesity and overweight: a systematic review and meta-analysis. BMC Public Health. 2009;9:88.

3. Whitlock G, Lewington S, Sherliker P, et al. Body-mass index and cause-specific mortality in 900,000 adults: collaborative analyses of 57 prospective studies. Lancet. 2009;373:1083-96.

4. Aune D, Sen A, Prasad M, et al. BMI and all cause mortality: systematic review and non-linear dose-response meta-analysis of 230 cohort studies with 3.74 million deaths among 30.3 million participants. BMJ. 2016;353:i2156.

5. Global BMI Mortality Collaboration. Body-mass index and allcause mortality: individual-participant-data meta-analysis of 239 prospective studies in four continents. Lancet. 2016;388:776-86.

6. Aune D, Sen A, Norat T, et al. Body mass index, abdominal fatness and heart failure incidence and mortality: a systematic review and dose-response meta-analysis of prospective studies. Circulation. 2016;133:639-49.

7. World Cancer Research Fund/American Insitute for Cancer Research. Food, nutrition, physical activity and the prevention of cancer: a global perspective. Washington: AICR; 2007.
8. Aune D, Greenwood DC, Chan DS, et al. Body mass index, abdominal fatness and pancreatic cancer risk: a systematic review and non-linear dose-response meta-analysis of prospective studies. Ann Oncol. 2012;23:843-52.

9. Aune D, Navarro Rosenblatt DA, Chan DS, et al. Anthropometric factors and ovarian cancer risk: a systematic review and nonlinear dose-response meta-analysis of prospective studies. Int J Cancer. 2014;136:1888-98.

10. Aune D, Navarro Rosenblatt DA, Chan DS, et al. Anthropometric factors and endometrial cancer risk: a systematic review and dose-response meta-analysis of prospective studies. Ann Oncol. 2015;26:1635-48.

11. Malik VS, Willett WC, Hu FB. Global obesity: trends, risk factors and policy implications. Nat Rev Endocrinol. 2013;9:13-27.

12. Benjamin EJ, Blaha MJ, Chiuve SE, et al. Heart disease and stroke statistics-2017 update: a report from the American Heart Association. Circulation. 2017; 135:e146-603.

13. Stecker EC, Vickers C, Waltz J, et al. Population-based analysis of sudden cardiac death with and without left ventricular systolic dysfunction: two-year findings from the Oregon Sudden Unexpected Death Study. J Am Coll Cardiol. 2006;47:1161-6.

14. Aune D, Sen A, Schlesinger S, et al. Body mass index, abdominal fatness, fat mass and the risk of atrial fibrillation: a systematic review and dose-response meta-analysis of prospective studies. Eur J Epidemiol. 2017;31:181-92.

15. Wannamethee G, Shaper AG, Macfarlane PW, Walker M. Risk factors for sudden cardiac death in middle-aged British men. Circulation. 1995;91:1749-56.

16. Jouven X, Desnos M, Guerot C, Ducimetiere P. Predicting sudden death in the population: the Paris Prospective Study I. Circulation. 1999;99:1978-83.

17. Kataoka M, Ito C, Sasaki H, Yamane K, Kohno N. Low heart rate variability is a risk factor for sudden cardiac death in type 2 diabetes. Diabetes Res Clin Pract. 2004;64:51-8.

18. Chei CL, Iso H, Yamagishi K, Inoue M, Tsugane S. Body mass index and weight change since 20 years of age and risk of coronary heart disease among Japanese: the Japan Public Health Center-Based Study. Int J Obes (Lond). 2008;32:144-51.

19. Laukkanen JA, Makikallio TH, Rauramaa R, Kiviniemi V, Ronkainen K, Kurl S. Cardiorespiratory fitness is related to the risk of sudden cardiac death: a population-based follow-up study. J Am Coll Cardiol. 2010;56:1476-83.

20. Ohira T, Maruyama M, Imano H, et al. Risk factors for sudden cardiac death among Japanese: the Circulatory Risk in Communities Study. J Hypertens. 2012;30:1137-43.

21. Bertoia ML, Allison MA, Manson JE, et al. Risk factors for sudden cardiac death in post-menopausal women. J Am Coll Cardiol. 2012;60:2674-82.

22. Lahtinen AM, Noseworthy PA, Havulinna AS, et al. Common genetic variants associated with sudden cardiac death: the FinSCDgen study. PLoS ONE. 2012;7:e41675.

23. Karppi J, Laukkanen JA, Makikallio TH, Ronkainen K, Kurl S. Serum beta-carotene and the risk of sudden cardiac death in men: a population-based follow-up study. Atherosclerosis. 2013;226:172-7.

24. Laukkanen JA, Makikallio TH, Ronkainen K, Karppi J, Kurl S. Impaired fasting plasma glucose and type 2 diabetes are related to the risk of out-of-hospital sudden cardiac death and all-cause mortality. Diabetes Care. 2013;36:1166-71.

25. Chiuve SE, Sun Q, Sandhu RK, et al. Adiposity throughout adulthood and risk of sudden cardiac death in women. JACC Clin Electrophysiol. 2015;1:520-8.

26. Adabag S, Huxley RR, Lopez FL, et al. Obesity related risk of sudden cardiac death in the atherosclerosis risk in communities study. Heart. 2015;101:215-21. 
27. Eranti A, Aro AL, Kerola T, et al. Body mass index as a predictor of sudden cardiac death and usefulness of the electrocardiogram for risk stratification. Am J Cardiol. 2016;117:388-93.

28. Stroup DF, Berlin JA, Morton SC, et al. Meta-analysis of observational studies in epidemiology: a proposal for reporting. Meta-analysis Of Observational Studies in Epidemiology (MOOSE) group. JAMA. 2000;283:2008-12.

29. Wells G, Shea B, O'Connell D. et al. The Newcastle-Ottawa Scale (NOS) for assessing the quality of nonrandomised studies in meta-analyses. http://www.ohri.ca/programs/clinical_epide miology/oxford.asp. Accessed 15 Aug 2017

30. DerSimonian R, Laird N. Meta-analysis in clinical trials. Control Clin Trials. 1986;7:177-88.

31. Greenland S, Longnecker MP. Methods for trend estimation from summarized dose-response data, with applications to meta-analysis. Am J Epidemiol. 1992;135:1301-9.

32. Bagnardi V, Zambon A, Quatto P, Corrao G. Flexible meta-regression functions for modeling aggregate dose-response data, with an application to alcohol and mortality. Am J Epidemiol. 2004;159:1077-86.

33. Higgins JP, Thompson SG. Quantifying heterogeneity in a metaanalysis. Stat Med. 2002;21:1539-58.

34. Egger M, Davey SG, Schneider M, Minder C. Bias in metaanalysis detected by a simple, graphical test. BMJ. 1997;315:629-34.

35. Begg CB, Mazumdar M. Operating characteristics of a rank correlation test for publication bias. Biometrics. 1994;50:1088-101.

36. Carter BD, Abnet CC, Feskanich D, et al. Smoking and mortality-beyond established causes. N Engl J Med. 2015;372:631-40.

37. Winslow UC, Rode L, Nordestgaard BG. High tobacco consumption lowers body weight: a Mendelian randomization study of the Copenhagen General Population Study. Int J Epidemiol. 2015;44:540-50.

38. Rimm EB, Stampfer MJ, Colditz GA, Chute CG, Litin LB, Willett WC. Validity of self-reported waist and hip circumferences in men and women. Epidemiology. 1990;1:466-73.

39. Bes-Rastrollo M, Sabate J, Jaceldo-Siegl K, Fraser GE. Validation of self-reported anthropometrics in the Adventist Health Study 2. BMC Public Health. 2011;11:213.

40. Weaver TW, Kushi LH, McGovern PG, et al. Validation study of self-reported measures of fat distribution. Int J Obes Relat Metab Disord. 1996;20:644-50.

41. Spencer EA, Appleby PN, Davey GK, Key TJ. Validity of selfreported height and weight in 4808 EPIC-Oxford participants. Public Health Nutr. 2002;5:561-5.

42. Flegal KM, Shepherd JA, Looker AC, et al. Comparisons of percentage body fat, body mass index, waist circumference, and waist-stature ratio in adults. Am J Clin Nutr. 2009;89:500-8.

43. Blew RM, Sardinha LB, Milliken LA, et al. Assessing the validity of body mass index standards in early postmenopausal women. Obes Res. 2002;10:799-808.

44. Dudina A, Cooney MT, Bacquer DD, et al. Relationships between body mass index, cardiovascular mortality, and risk factors: a report from the SCORE investigators. Eur J Cardiovasc Prev Rehabil. 2011;18:731-42.

45. Abdullah A, Peeters A, de Courten M, Stoelwinder J. The magnitude of association between overweight and obesity and the risk of diabetes: a meta-analysis of prospective cohort studies. Diabetes Res Clin Pract. 2010;89:309-19.

46. Cupples LA, Gagnon DR, Kannel WB. Long- and short-term risk of sudden coronary death. Circulation. 1992;85:I11-8.

47. Kannel WB, Cupples LA, D'Agostino RB. Sudden death risk in overt coronary heart disease: the Framingham Study. Am Heart J. 1987;113:799-804.
48. Cuspidi C, Rescaldani M, Sala C, Grassi G. Left-ventricular hypertrophy and obesity: a systematic review and meta-analysis of echocardiographic studies. J Hypertens. 2014;32:16-25.

49. Brady TM. The role of obesity in the development of left ventricular hypertrophy among children and adolescents. Curr Hypertens Rep. 2016;18:3.

50. Falkner B, DeLoach S, Keith SW, Gidding SS. High risk blood pressure and obesity increase the risk for left ventricular hypertrophy in African-American adolescents. J Pediatr. 2013;162:94-100.

51. Diez J. Effects of aldosterone on the heart: beyond systemic hemodynamics? Hypertension. 2008;52:462-4.

52. Rossi RC, Vanderlei LC, Goncalves AC, et al. Impact of obesity on autonomic modulation, heart rate and blood pressure in obese young people. Auton Neurosci. 2015;193:138-41.

53. Strandheim A, Halland H, Saeed S, et al. Obesity-associated metabolic changes influence resting and peak heart rate in women and men. Scand Cardiovasc J. 2015;49:337-43.

54. Aune D, Sen A, O'Hartaigh B, et al. Resting heart rate and the risk of cardiovascular disease, total cancer, and all-cause mortality-a systematic review and dose-response meta-analysis of prospective studies. Nutr Metab Cardiovasc Dis. 2017;27:504-17.

55. Messerli FH. Cardiovascular effects of obesity and hypertension. Lancet. 1982;1:1165-8.

56. Plourde B, Sarrazin JF, Nault I, Poirier P. Sudden cardiac death and obesity. Expert Rev Cardiovasc Ther. 2014;12:1099-110.

57. Ouchi N, Kihara S, Funahashi T, Matsuzawa Y, Walsh K. Obesity, adiponectin and vascular inflammatory disease. Curr Opin Lipidol. 2003;14:561-6.

58. Wong C, Marwick TH. Obesity cardiomyopathy: pathogenesis and pathophysiology. Nat Clin Pract Cardiovasc Med. 2007;4:436-43.

59. Joshy G, Korda RJ, Attia J, Liu B, Bauman AE, Banks E. Body mass index and incident hospitalisation for cardiovascular disease in 158,546 participants from the 45 and Up Study. Int J Obes (Lond). 2014;38:848-56.

60. Danaei G, Lu Y, Singh GM, Carnahan E, Stevens GA, Cowan MJ, Farzadfar F, Lin JK, Finucane MM, Rao M, Khang YH. Cardiovascular disease, chronic kidney disease, and diabetes mortality burden of cardiometabolic risk factors from 1980 to 2010: a comparative risk assessment. Lancet Diabetes Endocrinol. 2014;2:634-47.

61. Odutayo A, Wong CX, Hsiao AJ, Hopewell S, Altman DG, Emdin CA. Atrial fibrillation and risks of cardiovascular disease, renal disease, and death: systematic review and meta-analysis. BMJ. 2016;354:i4482.

62. Messerli FH, Nunez BD, Ventura HO, Snyder DW. Overweight and sudden death. Increased ventricular ectopy in cardiopathy of obesity. Arch Intern Med. 1987;147:1725-8.

63. Bigger JT Jr, Fleiss JL, Kleiger R, Miller JP, Rolnitzky LM. The relationships among ventricular arrhythmias, left ventricular dysfunction, and mortality in the 2 years after myocardial infarction. Circulation. 1984;69:250-8.

64. Anthony R, Daubert JP, Zareba W, et al. Mechanisms of ventricular fibrillation initiation in MADIT II patients with implantable cardioverter defibrillators. Pacing Clin Electrophysiol. 2008;31:144-50.

65. Bharati S, Lev M. Cardiac conduction system involvement in sudden death of obese young people. Am Heart J. 1995;129:273-81.

66. Balsaver AM, Morales AR, Whitehouse FW. Fat infiltration of myocardium as a cause of cardiac conduction defect. Am J Cardiol. 1967;19:261-5. 
67. Frank S, Colliver JA, Frank A. The electrocardiogram in obesity: statistical analysis of 1,029 patients. J Am Coll Cardiol. 1986;7:295-9.

68. Alpert MA, Terry BE, Cohen MV, Fan TM, Painter JA, Massey $\mathrm{CV}$. The electrocardiogram in morbid obesity. Am J Cardiol. 2000;85(908-10):A10.

69. O'Neal WT, Singleton MJ, Roberts JD, et al. Association between QT-interval components and sudden cardiac death: the ARIC Study (Atherosclerosis Risk in Communities). Circ Arrhythm Electrophysiol. 2017;10:e005485.

70. Reinier K, Narayanan K, Uy-Evanado A, et al. Electrocardiographic markers and the left ventricular ejection fraction have cumulative effects on risk of sudden cardiac death. JACC Clin Electrophysiol. 2015;1:542-50.
71. Aro AL, Reinier K, Rusinaru C, et al. Electrical risk score beyond the left ventricular ejection fraction: prediction of sudden cardiac death in the Oregon Sudden Unexpected Death Study and the Atherosclerosis Risk in Communities Study. Eur Heart J. 2017;38:3017-25.

72. Laukkanen JA, Di AE, Khan H, Kurl S, Ronkainen K, Rautaharju P. T-wave inversion, QRS duration, and QRS/T angle as electrocardiographic predictors of the risk for sudden cardiac death. Am J Cardiol. 2014;113:1178-83.

73. Alpert MA, Terry BE, Hamm CR, et al. Effect of weight loss on the ECG of normotensive morbidly obese patients. Chest. 2001;119:507-10. 